\section{The Supplementary Examination of the Rehabilitation by the Program Development to the Disabled Patients}

Takizawa, Shigeo ${ }^{1}$, Rika Wada ${ }^{2}$, Toshihiro Tachibana ${ }^{2}$ 'Biophilia Institute of Rehabiliaid Inc. Fujisawa, 2510871 ${ }^{2}$ Tachibana Orthopedic Surgery, Kobe, 6550041

We report supplementary examination of the Takizawa program. We enforced it at home rehabilitation by the program that we have confirmed the function improvement as 21 people in 35 who had been evaluated with apraxia of gait of the 49 evaluation targets had re-acquired walking with the use of any device by the research with the national grant by the physicians and physical therapist ${ }^{(1)}$.

The enforcement was conformed by the control system of the program. This program is usually enforced individually by Ms. Kyoko Takizawa.

We enforced the same program under the condition that she could not give any influence because the place where we enforced was beyond $500 \mathrm{Km}$ for her. The evaluation targets of us were 3 women. (1) Hemiplegia in the right and aphasia by the cerebral hemorrhage ( 67 years old) improved the degree of care for the point from 4 to 3. (2) Parkinson's disease (70) improved 11 points (87a 98); and (3) Parkinsonian disease and the post-operative of femoral head replacement (74) improved 13 points (83a 96) in the modified Functional Independent Measure.

We demonstrate that the Takizawa program was effective for home rehabilitation in three examples and available with any physical therapist.

\section{Reference:}

1) Yoshiko Morita, Kimura T. et all: The research for the examination of the ADL improvement of the lower bone fracture and to live independently for the disabled elderly. Civil publication. Fujisawa.31-36.

This research was partially supported by the Ministry of Education, Science, Sports and Culture, Grant-in-Aid for the research on the rehabilitation program diffusion toward the reduction of bedridden elderly.

\section{Changes in the Debilitated Elderly by Takizawa Rehabilitation Program}

Mitsuyo Makita Prof.(R.P.T.)

Department of Physical School of Health Science Niigata University of Health and Welfare

1398 Shimami-cho Niigata, 950-3198, Japan

\section{Purpose}

Takizawa rehabilitation program is carried out to the bedridden inpatients with the health care facility for the elderly by the physical therapist, where is occupied the bedridden in the majority. Improvements of the transfer and toilet activities were confirmed.

\section{Method}

I investigated it with the rehabilitation record in back watch and analyzed a change of the place and investigation time by using the sign test.

\section{Results}

(1) 47 people ( 17 men and 30 women), average $86.1 \pm 8.83$ years old (56-103 years old) have been receiving rehabilitation and the average placed days until the investigation were 365-day (25-904 days) at the investigation moment.

(2) 8 independences (direct assist is unnecessary, but help by the voice is necessary.) became 16 people at the investigation moment in the standing position and the transfer.

(3) At the placed time, though there were 23 diaper users, who decreased to 13 people at the investigation moment.

(4) The debilitated elderly inpatients who used diaper on admission to the facility, excreted in the rest room. And also the degree of the toilet activities is improved.

( sign test, $\mathrm{p} \leq 0.01$ ) 